\title{
Chapter 8 \\ Remediation Technology For Cesium Using Microbubbled Water Containing Sodium Silicate
}

\author{
Yoshikatsu Ueda, Yomei Tokuda, and Hiroshi Goto
}

\begin{abstract}
Remediation of materials contaminated with a radioactive material such as ${ }^{137} \mathrm{Cs}$ is important for public health and environmental concerns. Here, we report the effectiveness of aqueous sodium metasilicate (SMC) prepared using a microbubble crushing process for the removal of radioactive ${ }^{137} \mathrm{Cs}$ from contaminated materials. We have already reported that almost $80 \%{ }^{137} \mathrm{Cs}$ removal was achieved for a nonwoven cloth sample in which multiple washings using low SMC concentrations were effective. In addition, the volume of the waste solution can be reduced by neutralizing the SMC and using gelation to remove the radioactive material. We also attempt to clarify the mechanism of SMC operation by measuring its electrical properties. Decontamination is shown to be more efficient with SMC than with sodium hydroxide, even for washing granule conglomerates.
\end{abstract}

Keywords Radioactive cesium • Microbubble • Sodium metasilicate • Cs-137

\subsection{Introduction}

The accident at the Fukushima Daiichi nuclear power plant in 2011 following the Great East Japan Earthquake resulted in the dispersal of radioactive Cs into the environment and the contamination of an extensive area of soil. Various decontamination methods have been developed and applied in Fukushima Prefecture [1-4], but optimum methods for remediation of materials under specific contamination conditions still need to be developed. Radioactive Cs adsorbs on soil particles through ion exchange with potassium $[5,6]$. Our research focused on using aqueous

\footnotetext{
Y. Ueda $(\bowtie)$

Research Institute for Sustainable Humanosphere, Kyoto University, Gokasho, Uji,

Kyoto 611-0011, Japan

e-mail: yueda@rish.kyoto-u.ac.jp

Y. Tokuda

Institute for Chemical Research, Kyoto University, Gokasho, Uji, Kyoto 611-0011, Japan

e-mail: tokuda@noncry.kuicr.kyoto-u.ac.jp

H. Goto

Kureha Trading Co., Ltd., 1-2-10, Horidomecho, Nihonbashi, Chuo-ku, Tokyo 103-0012, Japan
} 
Fig. 8.1 Photographs of aqueous sodium metasilicate irradiated by a green laser after storage for one-half year. The metasilicate was dissolved in water (a) without crushing, and (b) with crushing [1]. The black object in the lower right corner is the green laser

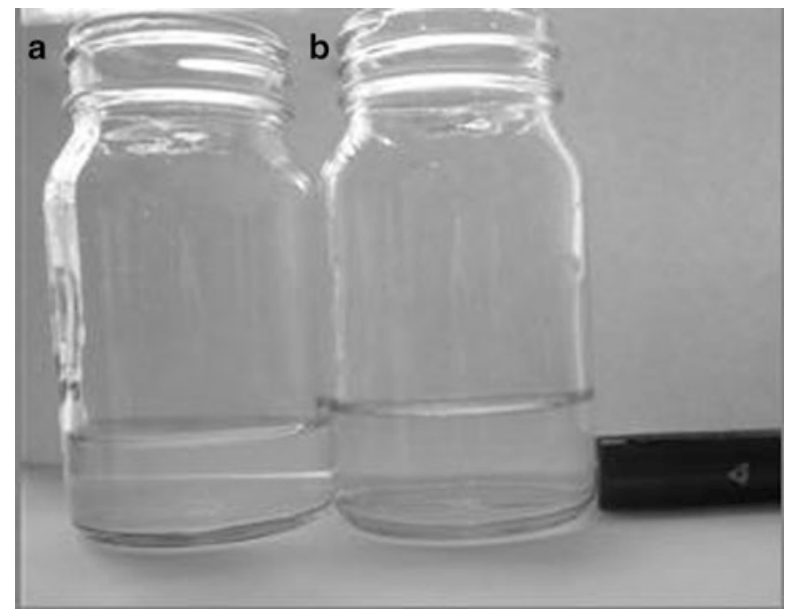

sodium metasilicate as a new detergent and possible decontamination agent. We examined the mechanism and principle of the change in the chemical reaction characteristics of aqueous sodium metasilicate after microbubble and ultrasonic treatments. We found that, because aqueous sodium metasilicate is not a surfactant, it has a low environmental load and does not exhibit foaming characteristics. It can be used with hard, soft, or sea water and is a "peeling detergent" in terms of its cleaning activity, which differentiates it from dissolving detergents such as organic solvents. As a peeling detergent, aqueous sodium metasilicate is suitable for foaming, jet streaming, high-pressure ultrasonic wave, and spray cleaning. A microbubble crushing process $[7,8]$ can also be used to suppress precipitation during long-term storage [9] (see Fig. 8.1).

Coprecipitation can be used to decrease the volume of the waste solution produced because sodium metasilicate turns into a gel that traps Cs when neutralized with acid [10]. Coprecipitation followed by gel formation can be used to separate the ${ }^{137} \mathrm{Cs}$ gel from the soil, yielding a decontaminated waste product.

\subsection{Experimental}

\subsubsection{Preparation of Aqueous Sodium Metasilicate}

A $0.47 \mathrm{~mol} / \mathrm{kg}$ solution of sodium metasilicate nonahydrate $\left(\mathrm{Na}_{2} \mathrm{O}_{3} \mathrm{Si} \bullet 9 \mathrm{H}_{2} \mathrm{O}\right)$ was prepared by dissolution in filtered water (manufactured using G-20B; Organo Corporation). A microbubble generator (capable of generating $>20,000$ microbubbles/mL) was manufactured by Kyowa Engineering and used to interfuse microbubbles into the solution. Ultrasonic irradiation $(40 \mathrm{kHz}$; ultrasonic wave generator UT-1204U and ultrasonic transducer UI-12R3; Sharp Corporation) was employed to crush the bubbles, and the resulting solution, aqueous sodium metasilicate prepared 
with a microbubble crushing process (abbreviated here as SMC), was used as the nonsurfactant aqueous detergent. Purified water and aqueous sodium hydroxide with the same $\mathrm{pH}$ as SMC $(\mathrm{pH}=13.1)$ were prepared for comparison. To analyze the structure of the sodium metasilicate in SMC and understand the dissolution stability, we measured the electrical conductivity and dissolved oxygen content every 5 min during the microbubble crushing process. We also compared the change in conductivity of SMC after $6 \mathrm{~h}$.

\subsubsection{Cleaning Method}

Depending on the individual diameters and buoyancy, microbubbles only remain in water for several minutes. They can remain in an aqueous environment for longer times, however, if an ultrasound treatment is used to reduce their size [11]. An ultrasound pretreatment of aqueous sodium metasilicate to form SMC and reduce the bubble size does not decrease the eduction rate of aqueous sodium metasilicate and offers the possibility of sustained cleansing. In this experiment, we conducted a cleaning test in a standing solution so that we could focus on the chemical cleansing effects due to the synergy between aqueous sodium metasilicate and microbubbles as opposed to focusing on the physical cleansing effects [1].

Granule conglomerates and nonwoven cloths were used as the materials to be cleaned. The $\sim 150$-g granule conglomerate samples were collected from soil in a hot spot $(\sim 500,000 \mathrm{~Bq} / \mathrm{kg})$ at the Fukushima Agricultural Technology Centre. These pieces of nonwoven cloth (made of polypropylene) were used in farm work at farms exposed to the fallout from the nuclear accident in Fukushima Prefecture. The cloths had an average weight of $2.65 \mathrm{~g}$, and the average amount of ${ }^{137} \mathrm{Cs}$ exhibited approximately $1633 \mathrm{~Bq} / \mathrm{sample}(616,226 \mathrm{~Bq} / \mathrm{kg})$ of radioactivity.

The granule conglomerate and nonwoven cloth samples were immersed in $100 \mathrm{~mL}$ of several SMC concentrations for $20 \mathrm{~h}$. Twelve granule conglomerate samples were prepared. These samples were divided into four groups of three samples. One group was washed in $\mathrm{NaOH}$, one in normal sodium metasilicate, and the remaining two were washed in 10- and 100-wt\% solutions of SMC. The 28 cloth samples were divided into 4 groups of 7 samples, and 1 group was washed in water, while the other 3 groups were washed in 1-, 10-, and 100-wt\% solutions of SMC. To examine the effects of multiple washings, samples were tested after $6 \mathrm{~h}$ of immersion before the second and third washes. After these immersion tests, the samples were dried at $40{ }^{\circ} \mathrm{C}$ for $40 \mathrm{~h}$ until they were free of moisture [2].

To reduce the volume of waste solution, hydrochloric acid $(\mathrm{HCl})$ was added to neutralize the detergent solutions after washing, which resulted in gelation. The gel and clear supernatant were then separated by filtration, and the radiation intensities (counts per second, cps) of the gel and supernatant were measured. We tried to remove ${ }^{137}$ Cs from SMC solutions by neutralization. We prepared three samples of 10 and $100 \mathrm{wt} \%$ SMC solutions, and then neutralized and filtered them. We measured the radiation intensity and compared the results of the two remediation methods. 


\subsubsection{Radiation Measurement}

The background radiation intensity of the nonwoven cloth specimens was measured using the germanium semiconductor detector at the Radioisotope Research Center, Kyoto University. The main unit of the detector is made from high-purity germanium (GMX-18200-S; EG\&G Ortec), with a germanium crystal that is $102 \mathrm{~cm}^{3}$ and a relative efficiency of $22.3 \%$ (efficiency ratio of a $3^{\prime \prime} \times 3^{\prime \prime} \mathrm{NaI}$ (TI) $(76 \times 76 \mathrm{~mm})$ crystal relative to that of the ${ }^{137} \mathrm{Cs} 662 \mathrm{keV}$ gamma ray). The entrance window is a 0.5 -mm-thick beryllium plate, which allows the detection of X-rays $3 \mathrm{keV}$ or higher in energy, as well as high-energy gamma rays. The energy resolution was $0.54 \mathrm{keV}$ for ${ }^{55} \mathrm{Fe} 5.9-\mathrm{keV}(\mathrm{Mn} \mathrm{K} \alpha) \mathrm{X}$-rays and $1.8 \mathrm{keV}$ for ${ }^{60} \mathrm{Co} 133-\mathrm{MeV}$ gamma rays. A special container $(100 \mathrm{~mL})$ was used to analyze the nonwoven cloth specimens. The removal ratio was defined as the ratio of the radiation intensity from the sample after and before immersion, as shown in Eq. 8.1.

$$
\text { Removal ratio }[\%]=\frac{\text { After immersion }[\mathrm{cps}]}{\text { Before immersion }[\mathrm{cps}]} \times 100
$$

\subsection{Results}

\subsubsection{Electrical Properties of SMC}

Figure 8.2 shows the measured electrical conductivity and dissolved oxygen content during the SMC-making process. The electrical conductivity of each sample was measured every 5 min. In Fig. 8.3, we show real-time electrical conductivity data sampled $6 \mathrm{~h}$ after the data in Fig. 8.2. The results show that SMC is almost stabilized after $10 \mathrm{~min}$, but compared with the real-time data, $1 \mathrm{~h}$ of microbubble crushing was required to stabilize the electrical conductivity.

\subsubsection{Washing the Granule Conglomerate}

The removal ratios of ${ }^{137} \mathrm{Cs}$ from the granule conglomerate using SMC (10 and $100 \mathrm{wt} \%$ ), $\mathrm{NaOH}$, and aqueous sodium hydroxide (Fig. 8.4) show that, while the decontamination efficiency of SMC was low, it was still more than 10 times higher than using sodium hydroxide at the same $\mathrm{pH}$. Furthermore, the higher efficiency of SMC compared with sodium metasilicate without the microbubble treatment confirms the effectiveness of SMC. One of the characteristics of SMC is that the concentration of dissolved oxygen does not increase after aeration. Hence, a large number of adsorption pits (sites) are probably present, similar to zeolites. Thus, cesium ions present in water will be incorporated into the silicate. In addition, the 


$$
\begin{aligned}
& \text { - - Conductivity }(\mu \mathrm{S} / \mathrm{cm}) \\
& -\Delta-\mathrm{DO}(\mathrm{mg} / \mathrm{L})
\end{aligned}
$$

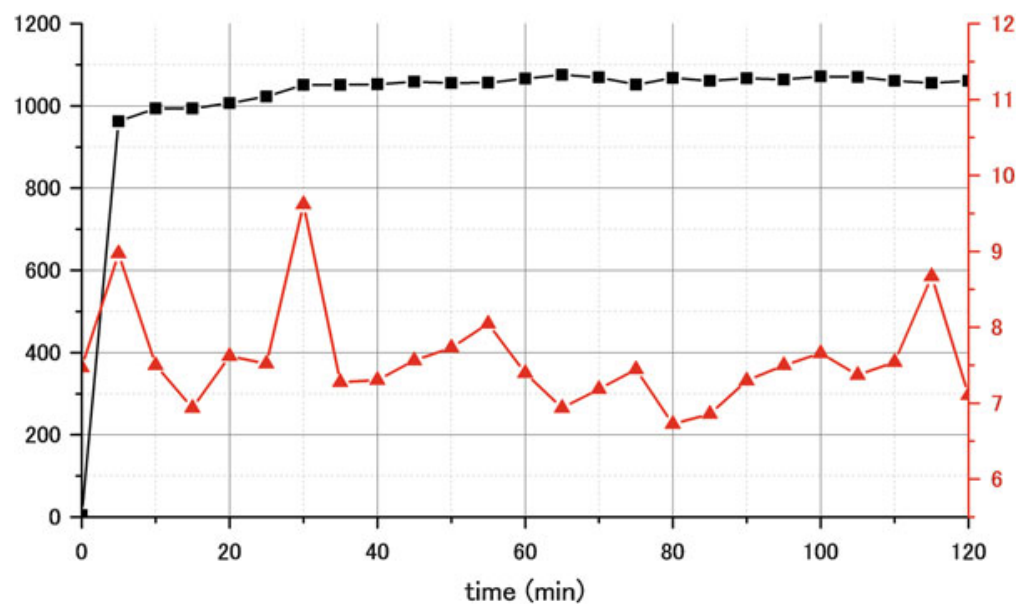

Fig. 8.2 Electrical conductivity and dissolved oxygen content during the microbubble crushing process

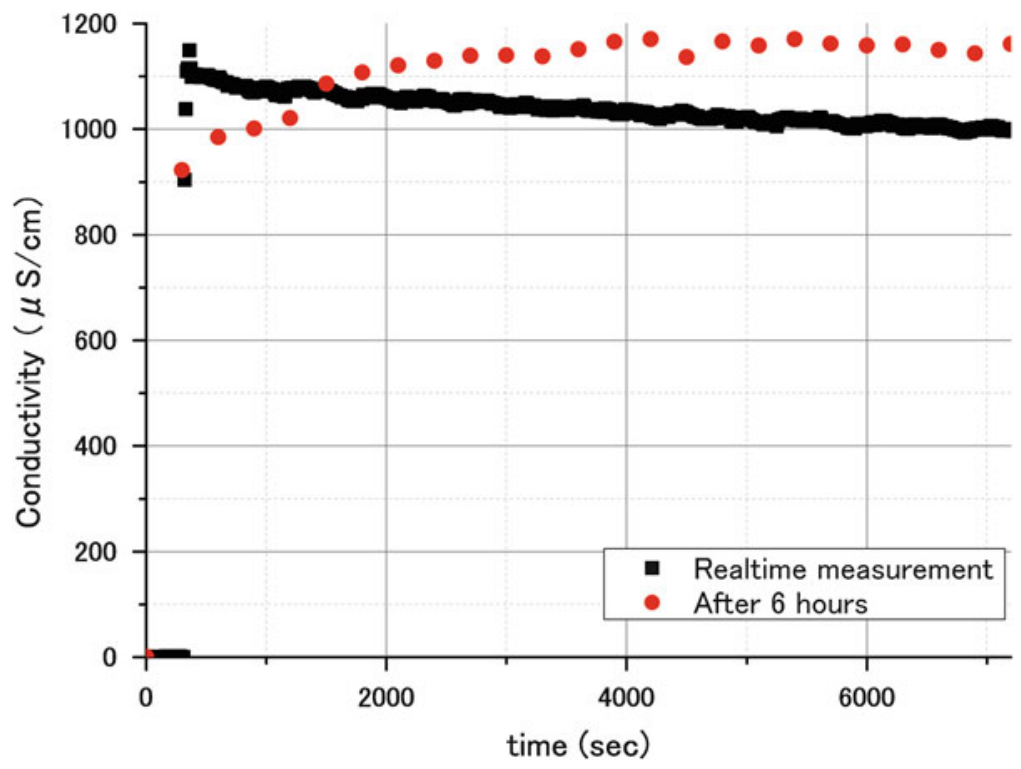

Fig. 8.3 Plot of conductivity versus time during the microbubble crushing process 


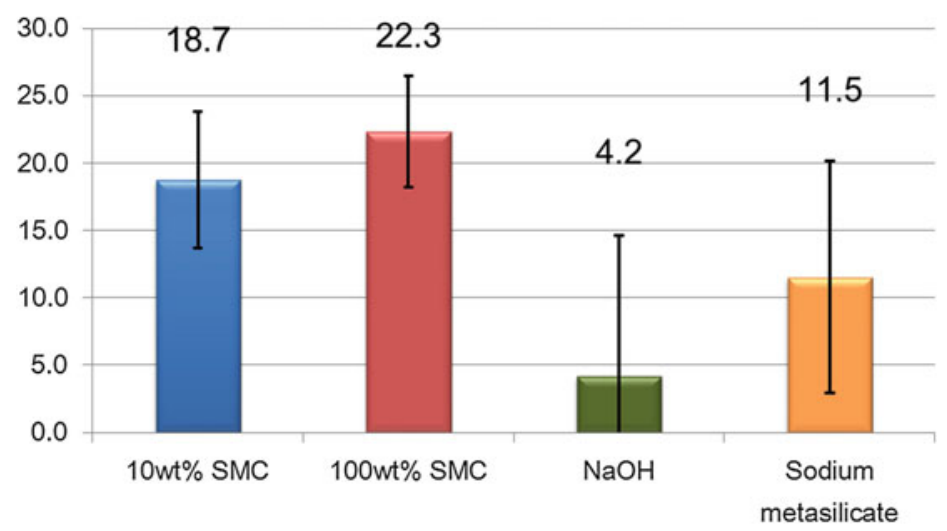

Fig. 8.4 Average removal ratios of ${ }^{137} \mathrm{Cs}$ from the granule conglomerate samples using 10- and $100-w t \%$ SMC, $\mathrm{NaOH}$, and sodium metasilicate

Table 8.1 ${ }^{137} \mathrm{Cs}$ removal ratios of using purified water and SMC for the nonwoven cloth samples*

\begin{tabular}{l|l|l|l|l}
\hline Washing & Pure water & $1-\mathrm{wt} \%$ SMC & $10-\mathrm{wt} \%$ SMC & $100-\mathrm{wt} \%$ SMC \\
\hline First & 0 & 44.5 & 59.5 & 77.5 \\
\hline Second & - & 34.5 & 27.5 & 51.4 \\
\hline Third & - & 20.6 & - & - \\
\hline
\end{tabular}

*Pure water was used for the first washing, whereas 10- and 100-wt\% SMCs were used for the second washing

decontamination efficiency increased because the alkaline solutions dissolved the granule conglomerate.

\subsubsection{Washing the Nonwoven Cloth}

The cleaning performance of SMC at various concentrations is compared in Table 8.1, which lists the average removal ratios for multiple washings with SMC concentrations of 1,10 , and $100 \mathrm{wt} \%$ compared with pure water [2].

As expected, almost no ${ }^{137} \mathrm{Cs}$ was removed by washing with pure water, but significant decontamination occurred as soon as SMC was introduced. Since the nonwoven cloth samples were previously used in agricultural fields, they also contained traces of fertilizers and other organic materials. As mentioned previously, sodium metasilicate detergent is used for washing because it has a capacity for breaking down organic materials via saponification, which is a result of its alkaline nature. We anticipate that we will be able to increase the efficiency of this detergent using microbubbles and ultrasound treatments. Organic components such as sebum and oil that are contaminated with ${ }^{137} \mathrm{Cs}$ are likely to be eluted from the nonwoven 
cloth owing to the alkalinity of SMC. The data in Table 8.1 also show that even with $1 \mathrm{wt} \%$ SMC, the removal ratio is $60 \%$ of that obtained with $100 \mathrm{wt} \%$ SMC after the first washing. This result shows that the amount of detergent used for remediation can be reduced to a low concentration.

In the case of multiple washings, the background radiation intensity decreased considerably after the third washing using a highly concentrated (10 or $100 \mathrm{wt} \%$ ) SMC solution. Therefore, a $1 \mathrm{wt} \%$ SMC solution was used for further analysis. The removal ratios after each wash with the $1 \mathrm{wt} \%$ solution, listed in Table 8.1, were $64 \%$ for the second wash and $71 \%$ for the third wash, indicating that even at a concentration of $1 \mathrm{wt} \%$, significant decontamination of nonwoven cloth materials can be achieved after multiple washings. Though there was only a slight decrease in the removal ratios compared with higher wt\% SMC solutions, we believe that using $1 \mathrm{wt} \% \mathrm{SMC}$ is an effective washing method for even higher levels of contaminants because the need for further decontamination of materials in the Fukushima Prefecture still exists.

\subsubsection{Remediation of ${ }^{137}$ Cs by Neutralizing SMC}

Reduction in the waste solution volume obtained after using SMC was also investigated. We prepare three samples each of 10 and $100 \mathrm{wt} \%$ SMC waste solution. Solutions were neutralized by $\mathrm{HCl}$ and the gels separated from the filtered solution. By measuring the radiation intensity then normalizing the readings, we were able to compare the gels and filtered solutions (Fig. 8.5). ${ }^{137} \mathrm{Cs}$ was captured

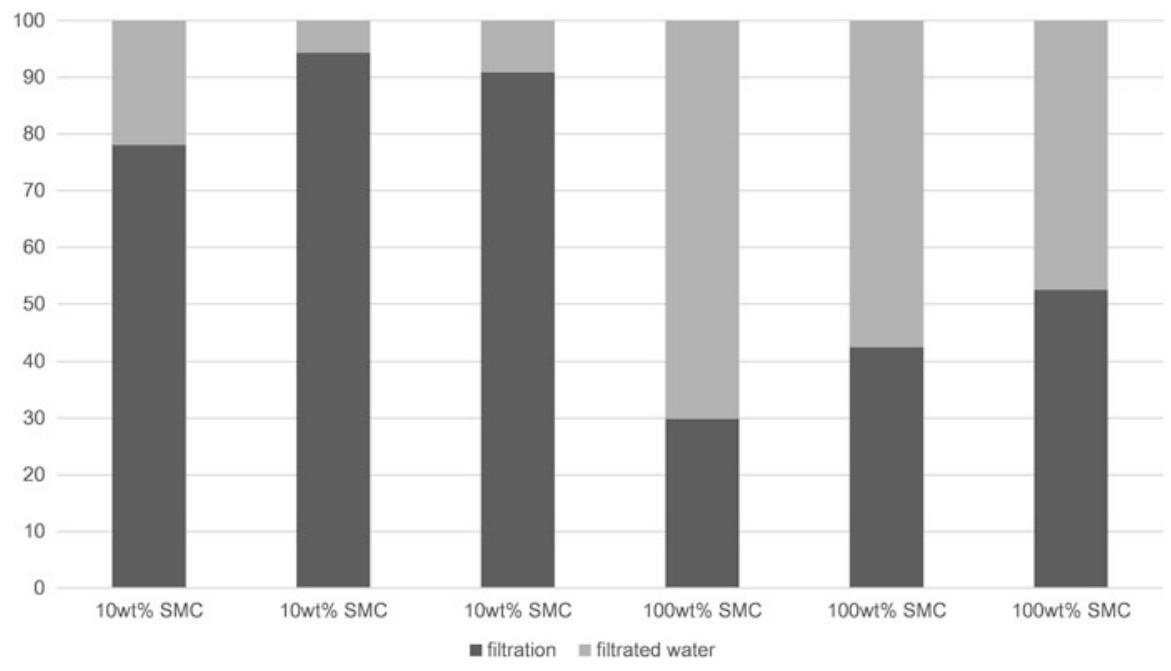

Fig. 8.5 Remediation of ${ }^{137}$ Cs by neutralizing SMC (10 and $100 \mathrm{wt} \%$ ) 
in the gel after coprecipitation [1, 2]. Hence, each concentration of SMC has the ability to capture Cs effectively. We discovered, however, that the $100 \mathrm{wt} \% \mathrm{SMC}$ solution had an opposite ability compared with the other solutions. It is because sodium metasilicate can leave its captured ions in water. We need much additional experimentation to determine the optimum conditions for capturing Cs in SMC gels. We are currently trying to neutralize the SMC by alcohol and cleanup ${ }^{137} \mathrm{Cs}$ from waste solutions.

\subsection{Conclusions}

We have examined the cleaning performance of SMC by comparing the radioactivity of waste solutions before and after washing contaminated granule conglomerates and nonwoven cloth samples. A study of the changes in the chemical characteristics of SMC was also undertaken. ${ }^{137} \mathrm{Cs}$ particulates attached to a nonwoven cloth sample were removed effectively by SMC cleaning. After cleaning, the remaining dissolved aqueous sodium metasilicate from the SMC had a better treatment capacity (after neutralization) than standard aqueous solutions. Thus, the use of SMC should contribute significantly to the decontamination work currently being undertaken in urban and rural areas where decontamination cannot be performed by using water alone. We are currently attempting to measure the chemical structure of SMC by SPring-8. We will be able to report the results of this new study in the near future.

Acknowledgments This work was financially supported by Kyoto University, The Japan Association of National Universities, Japan Association for Chemical Innovation (JACI), ITOCHU Foundation, and the Collaborative Research Program of Institute for Chemical Research, Kyoto University (No. 2013-63). We thank N. Nihei, S. Fujimura, T. Kobayashi, Y. Ono, M. Tosaki, and T. Minami for helpful discussions and experimental preparation. We appreciate the SMC samples (JPAL) provided by Kureha Trading Co. Ltd. The synchrotron radiation experiments were performed at the BL14B2 beamline of the SPring-8 facility with the approval of the Japan Synchrotron Radiation Research Institute (JASRI) (Proposal No. 2015A1662).

Open Access This chapter is distributed under the terms of the Creative Commons Attribution Noncommercial License, which permits any noncommercial use, distribution, and reproduction in any medium, provided the original author(s) and source are credited.

\section{References}

1. Ueda Y, Tokuda Y, Goto H, Kobayashi T, Ono Y (2014) Removal of radioactive Cs using aqueous sodium metasilicate with reduced volumes of waste solution. ECS Trans 58(19):35

2. Ueda Y, Tokuda Y, Goto H, Kobayashi T, Ono Y (2013a) Removal of radioactive Cs from nonwoven cloth with less waste solution using aqueous sodium metasilicate. J Soc Remed Radioact Contam Environ 1:191 
3. Ueda Y, Tokuda Y, Fujimura S, Nihei N, Oka T (2013b) Cesium transfer from granule conglomerate using water containing nano-sized air bubbles. ECS Trans 50(22):1

4. Ueda Y, Tokuda Y, Fujimura S, Nihei N, Oka T (2013c) Removal of radioactive Cs from gravel conglomerate using water containing air Bubbles. Water Sci Technol 67:996

5. Raskin I, Ensley BD (eds) (2000) Phytoremediation of toxic metals: using plants to clean up the environment. Wiley, New York

6. Willey N (ed) (2007) Phytoremediation: methods and reviews. Humana Press, Totowa

7. Agarwal A, Ng WJ, Liu Y (2011) Principle and applications of microbubble and nanobubble technology for water treatment. Chemosphere 84:1175

8. Stride E (2008) The influence of surface adsorption on microbubble dynamics. Philos Trans R Soc A 366:2103

9. Suwabe J (2010) Manufacturing method of a water-based cleaning agent. Japanese Patent (Kokai), 2010-1451

10. Merrill RC, Spencer RW (1950) Gelation of sodium silicate; effect of sulfuric acid, hydrochloric acid, ammonium sulfate, and sodium aluminate. J Phys Colloid Chem 54:806

11. Takahashi M, Kawamura T, Yamamoto Y, Ohnari H, Himuro S, Shakutsui H (2003) Effect of shrinking microbubble on gas hydrate formation. J Phys Chem B 107:2171 